\title{
Leishmaniasis in Saudi Arabia: Current situation and future perspectives
}

\author{
Elfadil Abass', Zainab Al-Hashem², \\ Lamya Zohair Yamani ${ }^{3}$
}

\begin{abstract}
Background and Objective: Leishmaniasis is endemic in Saudi Arabia with cases reported in many regions. This review refers to publications on leishmaniasis in Saudi Arabia and discusses issues related to parasite species, clinical manifestation and diagnosis.

Methods: This research was done at Imam Abdulrahman Bin Faisal University, Dammam, Saudi Arabia by systematic literature search on PubMed and Google Scholar databases from 1989 to 2018. Selection criteria included original articles reporting on visceral leishmaniasis $(\mathrm{VL})$ or cutaneous leishmaniasis $(\mathrm{CL})$ in Saudi Arabia.

Results: The search identified 16 eligible articles, six for VL and 10 for CL. VL was reported in areas known to be non-endemic. Leishmania donovani was the main cause for human VL while Leishmania infantum seemed to cause the disease in animals. Dogs were considered the main reservoir hosts and black rats (Rattus rattus) were potential hosts. VL mainly affected infants and young children. It is important to note that VL diagnosis was based on either invasive parasite detection procedures or serologically using indirect hemagglutination test. CL represented the most frequent clinical form with the main endemic foci reported in the South-West and Eastern regions. CL appeared to have no demographic or socioeconomic restriction; it affected both rural and urban citizens, with the majority occurring among farmers. Travelling was recognized as an important risk factor. Leishmania tropica and Leishmania major were recognized as the main causes for $\mathrm{CL}$.

Conclusion: This report summarizes the potential risks for VL and CL in Saudi Arabia in areas known to be non-endemic. There are substantial gaps in knowledge and practices in regard to leishmaniasis in Saudi Arabia, highlighting the need for more research and medical surveillance targeting the disease in humans and animals.
\end{abstract}

KEYWORDS: Epidemiology, Leishmaniasis, Saudi Arabia.

How to cite this:

doi: https://doi.org/10.12669/pjms.36.4.2121

Abass E, Al-Hashem Z, Yamani LZ. Leishmaniasis in Saudi Arabia: Current situation and future perspectives. Pak J Med Sci. 2020;36(4):836-842. doi: https://doi.org/10.12669/pjms.36.4.2121

This is an Open Access article distributed under the terms of the Creative Commons Attribution License (http://creativecommons.org/licenses/by/3.0), which permits unrestricted use, distribution, and reproduction in any medium, provided the original work is properly cited.

Correspondence:

Elfadil Abass,

Department of Clinical Laboratory Science,

College of Applied Medical Sciences,

Imam Abdulrahman Bin Faisal University,

P.O. Box 2435, Dammam,

Saudi Arabia.

E-mail: emabass@iau.edu.sa

* Received for Publication:

* Revision Received:

* Revision Accepted: *
December 19, 2019

February 18,2020

February 28, 2020

\section{INTRODUCTION}

Leishmaniasis is a vector-borne disease caused by different species belonging to the genus Leishmania. These species cause various clinical manifestations ranging in severity from selflimited cutaneous lesions to life-threatening visceral disease, including cutaneousleishmaniasis (CL), visceral leishmaniasis (VL), mucocutaneous leishmaniasis (MCL), and post kala-azar dermal leishmaniasis, PKDL. ${ }^{1}$ The disease is categorized 
into two types: zoonotic leishmaniasis (where wild and domestic animals are considered main reservoir hosts) and anthroponotic leishmaniasis (where humans are the source of the infection). Several domestic and wild mammalian hosts are involved in the transmission cycle of the disease, including certain rodents and dogs. ${ }^{1,2}$

$\mathrm{VL}$ is the most severe form of the disease. It is caused by various species of Leishmania in different endemic regions; L. donovani in Asia and East Africa and L. infantum in the Mediterranean area, Middle East, central Asia and America. It is the most severe form of the disease and still associated with high mortality. ${ }^{1}$ Laboratory findings include anemia, leukopenia, thrombocytopenia, hypoalbuminemia, and hypergammaglobulinemia. Kidney damage in VL is well-known and can appear as glomerulonephritis, acute or chronic renal disease. ${ }^{3,4}$

The VL suspicion is present if fever persists more than two weeks in the presence of splenomegaly in individuals living in or having visited known
VL-endemic areas. Clinical diagnosis is confirmed by various laboratory methods. These methods are based on detection of the parasite in aspirates collected from spleen, liver, bone marrow or lymph nodes. Sensitivity of these methods depends on the type of sample, being less sensitive for lymph nodes while more sensitive for spleen samples. ${ }^{2,5}$ Due to the invasive nature of sample collection and low sensitivity, new methodologies based on detection of specific Leishmania antibody are currently used. The direct agglutination test (DAT) using intact promastigote antigen and enzyme-linked immunosorbent assay (ELISA) using refined recombinant proteins of Leishmania are most common methods. These methods are used for detection of VL in humans and animal hosts with variable diagnostic accuracy in the various endemic regions. ${ }^{6}$

Worldwide, CL is the most common form of leishmaniasis, causing the greatest disease burden. It occurs across the Indian subcontinent, through the Mediterranean region and from Africa to America.

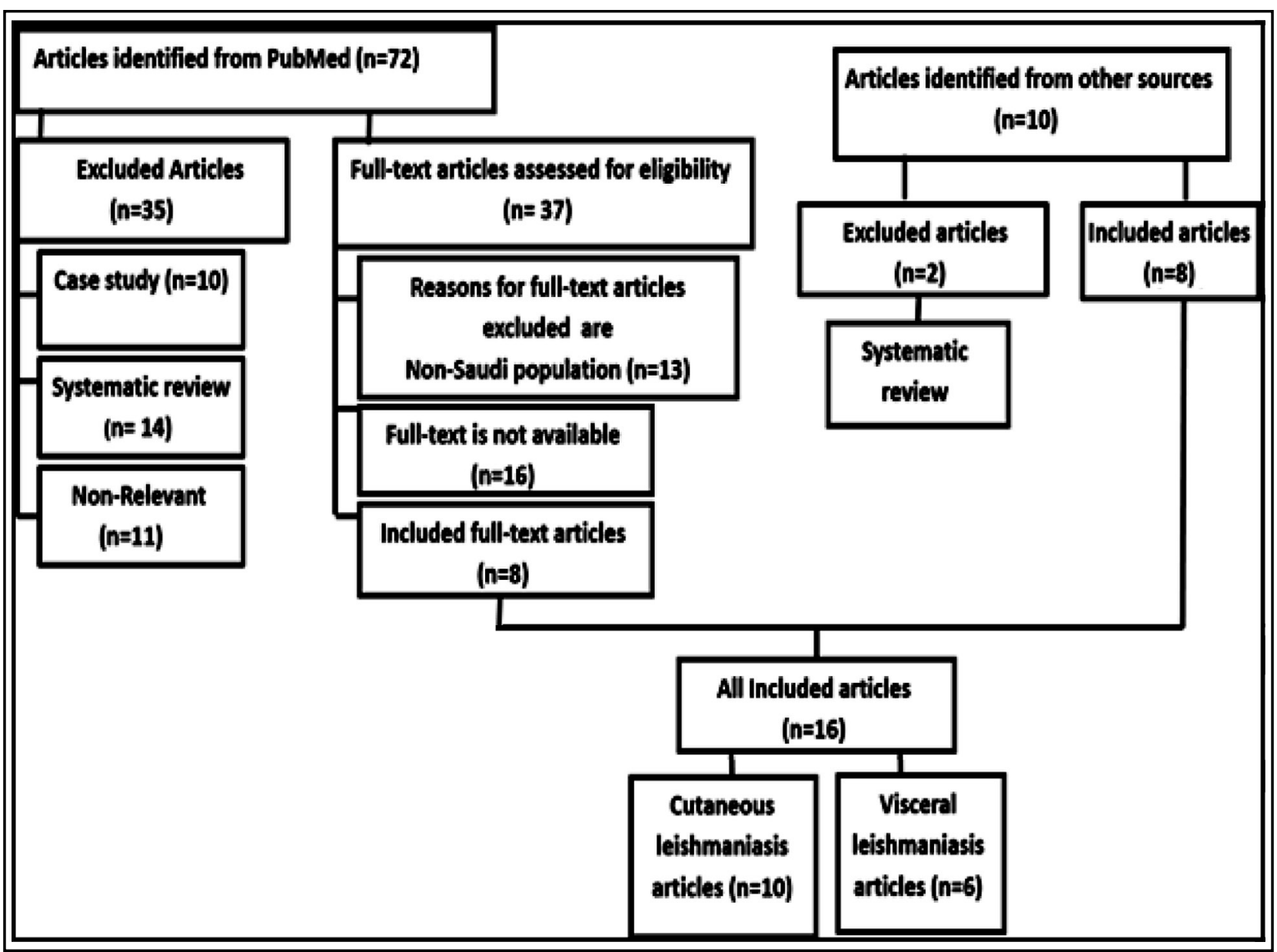

Fig.1: Flow chart of included and excluded scientific articles. 
Most of the cases occur in six countries including Afghanistan, Brazil, Colombia, Iran, Algeria and Syria. ${ }^{7.8}$

$\mathrm{CL}$ is a self-limiting skin disease, causing skin ulcers on the uncovered body parts at the place of the infected sand fly vector bite. The appearance of characteristic lesions in areas with high endemicity of CL is enough to establish the clinical diagnosis. However, laboratory tests are required to distinguish leishmaniasis from several other skin diseases. The diagnosis is classically based on direct detection of Leishmania in lesion smears stained by Giemsa-stain or by culture., ${ }^{9,10}$ PCR techniques are highly sensitive and help to determine the parasite species, but it requires painful and invasive procedures for sample collection. ${ }^{10,11}$ Serological diagnosis is frequently used in epidemiological studies of leishmaniasis. It is an easy and quick approach, but its sensitivity is low due to limited circulating antibodies and potential antigen diversity of parasites that cause the disease. . $^{12,13}$

\section{METHODS}

Literature Search Strategy: This systematic literature search was done at Imam Abdulrahman Bin Faisal University, Dammam, Saudi Arabia. It was conducted by a literature search identifying epidemiological studies reporting leishmaniasis in Saudi Arabia. The search was performed on electronic databases using the PubMed and Google Scholar. The search was performed from 1989-2018. Keywords that were used included: epidemiology, leishmaniasis and Saudi Arabia. Only original research articles written in English language were selected. Furthermore, a Google search was used as an additional source of data. ${ }^{14}$ These articles were also included in the data analysis.

\section{RESULTS}

Sixteen eligible articles were identified (Fig.1), six studies reported VL and 10 articles reported CL. Results showed an uneven distribution of both diseases in the Kingdom. It was not possible to count the total number of individuals infected by the disease. In most of the published studies, only selected cases were involved (Table-I and II). None of these studies reported outbreaks or co-infections with other diseases. There were no reports on parasite characterization and genetic heterogeneity. The role of black rats in epidemiology of the disease was not confirmed. Also, there were no reports in regard to usage of new laboratory tests for the disease confirmation. The published studies also lack information on the use of new leishmaniasis drugs/regimens.

\section{Visceral leishmaniasis:}

Epidemiological Characteristics: The major endemic areas that reported VL in Saudi Arabia were in the South and South-West. Sporadic cases were also reported in the city of Al-Madinah. Endemic areas included Gizan, Najran and Asser. In these areas, VL was characterized by seasonal variation with emergence of many cases in late spring and summer and few cases were observed in the winter. In Gizan, VL was confirmed in both humans and animals. L. donovani LON42 was associated with human infections while both $L$. donovani LON42 and L. infantum LON 49 were isolated from black rats (Rattus rattus). L. infantum LON49 was isolated only from dogs. In Najran, other animals might act as reservoirs for the parasite, such as goats and sheeps.

Most of the published articles reported VL among infant and adolescence in Gizan, Aseer and AlMadinah. There was no gender restriction; both male and females were equally affected. In a study carried out in Najran involving 384 human samples,

Table-I: Included studies of VL in Saudi Arabia.

\begin{tabular}{|c|c|c|c|c|}
\hline Author, Year [Reference] & Study Year & Study area & $\begin{array}{l}\text { Total } \\
\text { tified Cases }\end{array}$ & Diagnosis Method \\
\hline Mokhtar et al. $(2017)^{15}$ & NA & Najran, South West & 32 & IHAT \\
\hline Jamil.et.al. $(2012)^{16}$ & $1985-2007$ & Abha, South West & 123 & Parasite detection in bone marrow \\
\hline Jamil.et.al. $(2012)^{17}$ & $1985-2008$ & Aseer, South West & 582 & Parasite detection in bone marrow, IHAT \\
\hline Jack et al. $(2005)^{18}$ & 1988 & Al Madinah, West & 7 & Parasite detection in bone marrow, IHAT \\
\hline Al-Orainey et al. $(1994)^{19}$ & NA & Gizan, South West & 121 & $\begin{array}{l}\text { Parasite detection in liver/spleen, } \\
\text { culture, IHAT }\end{array}$ \\
\hline Ibrahim.et al. $(1992)^{20}$ & $1989-1990$ & Gizan, South West & NA & Parasite detection in liver/spleen, culture \\
\hline
\end{tabular}

IHAT: indirect hemagglutination assay test; NA: no available data. 
Elfadil Abass et al.

Table-II: Included Studies of CL in Saudi Arabia.

\begin{tabular}{|c|c|c|c|c|}
\hline Author, Year [Reference] & Study Year & Study area & $\begin{array}{c}\text { Total } \\
\text { Identified Cases }\end{array}$ & Diagnosis Method \\
\hline Hawash et al. $(2018)^{21}$ & 2016-2017 & Al-Taif, West & 90 & $\begin{array}{l}\text { Parasite detection in skin smears, } \\
\text { OligoC- PCR, and kDNA PCR }\end{array}$ \\
\hline Alahmad et al. $(2018)^{22}$ & $2009-2010$ & Al-Hassa, East & 901 & Parasite detection in skin smears \\
\hline Haouas et al. $(2017)^{23}$ & 2015-2017 & Hail, North West & 37 & $\begin{array}{l}\text { Parasite detection in skin smears } \\
\text { and SSU-rDNA- PCR }\end{array}$ \\
\hline Barradah $(2017)^{24}$ & $2007-2016$ & $\begin{array}{l}\text { Majmaah, } \\
\text { Riyadh Province }\end{array}$ & 87 & Parasite detection in skin smears \\
\hline Alanazi et al. $(2016)^{25}$ & $2009-2013$ & $\begin{array}{l}\text { Dawadim, } \\
\text { Riyadh Province }\end{array}$ & 370 & Parasite detection in skin smears \\
\hline Faraj \& Lake $(2014)^{26}$ & $1996-2007$ & Aseer, South West & NA & Parasite detection in skin smears \\
\hline El-Beshbishy et al. $(2013)^{27}$ & 2010-1012 & Al-Madinah, West & 34 & kDNA PCR \\
\hline Uthman et al. $(2005)^{28}$ & 1997-1999 & Al-Khobar, East & 120 & Parasite detection in skin smears \\
\hline Al-zahrani et al. (1989) ${ }^{29}$ & 1987 & $\begin{array}{l}\text { Al-Baha, } \\
\text { Aseer, West }\end{array}$ & 2302 & Parasite detection in skin smears \\
\hline Dye et al. $(1989)^{30}$ & 1986 & Al-Hassa, East & $284^{*}$ & $\begin{array}{l}\text { Identification of active lesions } \\
\text { and leishmanial scars }\end{array}$ \\
\hline
\end{tabular}

kDNA PCR: kinetoplast DNA polymerase chain reaction, SSU-rDNA PCR: small subunit ribosomal DNA PCR.

* The number included cases with active lesions (117) and leishmanial scars ( $n=167)$.

anti-Leishmania antibodies were significantly higher among old individuals.

Clinical Features: The common clinical features of VL among pediatric patients included fever $(100 \%)$, pallor $(>95 \%)$, hepatosplenomegaly $(>90 \%)$ and lymphadenopathy (>90\%). Other clinical presentations included abdominal distention, anorexia, and weight loss. However, none of the patients showed skin lesions (PKDL) while lymphadenopathy was a less common clinical finding. VL patients showed also abnormal laboratory results including anemia, leukopenia, thrombocytopenia, hypoalbuminemia and hypergammaglobulinemia. Abnormalities in liver functions were also observed.

Diagnostic Features: In endemic areas, primary VL suspected cases and diagnosis was based on clinical signs and symptoms. Laboratory diagnosis was based on either direct detection of Leishmania in bone marrow aspirates or serologically using indirect hemagglutination test (IHAT) kit (Siemens Healthcare Diagnostic, Marburg, Germany). IHAT detects anti-Leishmania antibodies in patients' sera with hemagglutination cutoff titers of 1: 32-1: 64 . This test performed very well with high sensitivity and specificity. Sensitivity of bone marrow aspiration for detection of Leishmania was also high $(92.7 \%)$. None of the studies detected Leishmania in lymph node aspirates. Some studies used splenic and liver aspirates and isolation of the parasite in diphasic media for confirmation of the disease.
Cutaneous leishmaniasis:

Epidemiological Characteristics: CL was endemic in various parts of Saudi Arabia, with most of the cases reported in East, West, South-West, NorthWest and the Southern regions. Endemic areas included Al-Hassa, Aseer, Al-Baha, Al-Madinah, Al-Taif and Hail. Several cases were also reported in areas known to be non-endemic such as Al-Khobar and Riyadh province. CL was more prevalent among male than female. Infection included all ages with infant and young adults being the majority affected. There was no socioeconomic restriction for the disease. The affected populations included both rural and urban citizens, mainly among farmers. Temperature was recognized as an important factor associated with CL transmission in Aseer. Travelling was also recognized as a risk factor. Studies confirmed L. tropica and L. major as the causative agents for the disease in Saudi Arabia. Various zymodemes of L. tropica were identified in the South-West region, including LON-71, LON-72, LON-73, LON-10 and LON-63. L. major zymodeme LON-1 and LON-4 were also reported in the region. Clinical Features: CL manifested as skin lesions on the face and exposed parts of the body. The number of skin lesions found in each patient differs, with the majority developing 2-3 lesions. In many cases the lesions were excoriated and were observed as insect bites. Ulcerative lesions varied in size (from $2-10 \mathrm{~cm}$ in diameter), with moderately rolled edges and granulomatous bases. The lesions were painful 
when associated with secondary bacterial infection and inflammation.

Diagnostic Features: Parasite culture and microscopic examination of smears were the most common laboratory methods for CL diagnosis. These methods were recommended to be used together for greater efficacy. Molecular tools such as PCR based on ITS1 and KDNA was used to facilitate the diagnosis and identification of the parasite. These methods found to be more sensitive than parasite culture and microscopic examination. They detected most of CL cases that were negative by direct examination methods. In a single study, kDNA PCR proved to be more sensitive than microscopy and OligoC-TesT PCR.

\section{DISCUSSION}

This study reports epidemiological data on the most common forms of leishmaniasis in Saudi Arabia. Although Saudi Arabia has experienced high prevalence of leishmaniasis, only minimal recent data has been published on this disease. Reporting updated information is crucial for planning any effective strategy for disease control. These strategies require detection of the disease in humans and animals, identification of reservoir hosts and sand fly vectors. ${ }^{31}$ The general department of the ministry of health in Saudi Arabia controls vector-borne and communicable disease has launched national policy for control of the disease in the country with a case notification system. This has led to marked reduction in the incidence of CL. ${ }^{32}$

In Saudi Arabia, most of the studies passively detected leishmaniasis with varying incidence rates in the different regions and among individuals that were originated from other countries. Estimation of the disease burden on basis of passive reporting would likely underestimate the magnitude of this disease, rendering control programs ineffective. These programs would have achieved better results through active case detection, through which asymptomatic or cases with atypical clinical presentations can be identified. Asymptomatic Leishmania infections are not well defined but are often serologically identified. Such asymptomatic infected hosts may serve as reservoirs, transmitting the disease silently. These reported cases are considered to be important reservoir hosts of the parasite, presenting an additional challenge in assessing Leishmania infection. It is important to note that asymptomatic Leishmania carrier individuals posses decreased antibody titers as compared to clinical VL cases. ${ }^{33}$ Indeed, seropositive individuals have increased chance to progress to symptomatic VL cases. However, substantial numbers of VL seropositive patients will never develop a clinical disease. In reality, it is hard to judge whether an apparently healthy seropositive individual is truly infected or not.

In recent years, important changes in epidemiology of leishmaniasis have been noticed. In addition to rural areas, leishmaniasis has become common in urban settings. Classically, VL affects adults that are more exposed to sand fly vectors. This classical picture has changed; the disease became more prevalent among infants and young children. ${ }^{34}$ This seems to be the case in Saudi Arabia, where VL has been reported in younger age groups. Indeed, exposure to Leishmania may lead to development of protective immunity. This could be the reason of the high prevalence of VL among infant and young children. It has been shown that, $\mathrm{VL}$ is more common among men than women due to the nature of their work. In Saudi Arabia, women have a special situation. They often stay indoors or cover their body when outdoor, leading to less exposure and thus less susceptibility to Leishmania.

Diagnosis of VL can be challenging due to the overlap of VL and other disease areas such as malaria, typhoid, tuberculosis, and HIV. These diseases can coincide with VL. Population movement and increased travelling have led to appearance of $\mathrm{VL}$ in areas that were previously free of the disease. In such cases, the patients may develop atypical clinical presentation and may remain undetected. Recently, Alwazzeh and Alhashimalsayed have reported a case of a 42-years old patient in AlKhobar (Eastern province), a known non-endemic VL area. ${ }^{4}$ This patient was originally from Gizan, but VL was not considered at first check-up due to atypical clinical presentation. After three months' delay, VL was diagnosed serologically at the Mayo Clinic Labs. This case could have been diagnosed earlier if serological tests were available.

CL has a wide distribution across the world, reaching from Asia, through Middle East and North Africa, to North-and South America. Saudi Arabia has the fourth highest prevalence rate of zoonotic CL after Afghanistan, Iran and Pakistan. It has been shown that factors such as poor access to health facilities along with the moderate nature of the disease (self-healing ulcers) have contributed to the poor passive reporting of the cases. $^{35}$ Therefore, the actual prevalence and incidence of the disease would be higher than 
reported previously. Also, accurate diagnostic test systems would help identification of these cases.

Molecular tools have identified L. major and L. tropica as causative agents of CL in Saudi Arabia, with the majority of the cases caused by L. major. L. tropica seems to be less prevalent, occurring within small foci in the west and southwest regions. ${ }^{23,27}$ Despite the high prevalence of the disease in the country, few studies have been focused on identification of Leishmania species and little is known about molecular and isoenzyme characterization of the parasites species. This review identified poor application of control tools, including unavailability of modern diagnostics and treatment regimens. In most of the previous studies, diagnosis was based on clinical examination and passive detection of the parasite in skin biopsies. Indeed, many skin conditions may display similar clinical features resembling $\mathrm{CL}$, thus laboratory diagnosis is essential. Additionally, considering the high toxicity and treatment cost, it is unacceptable to treat unconfirmed cases i.e., expose them to the medication.

\section{CONCLUSION}

This report highlights potential risks for VL and CL in Saudi Arabia in areas known to be non-endemic. There are also crucial gaps about diversity of the reservoir host and their role in the transmission cycle of Leishmania. We recommend active case detection using new test systems to identify the real number of undetected cases and to evaluate the magnitude of the problem.

\section{REFERENCES}

1. Burza S, Croft SL, Boelaert M. Leishmaniasis. Lancet. 2018;392:951-970. doi: 10.1016/S0140-6736(18)31204-2

2. Torres-Guerrero E, Quintanilla-Cedillo MR, RuizEsmenjaud J, Arenas R. Leishmaniasis: a review. F1000Res. 2017;6:750. doi: 10.12688/f1000research.11120.1

3. Tabbabi A. Review of Leishmaniasis in the Middle East and North Africa. Afr Health Sci. 2019;19(1):1329-1337. doi: 10.4314/ahs.v19i1.4

4. Alwazzeh MJ, Alhashimalsayed ZH. Visceral leishmaniasis and glomerulonephritis: A case report. Saudi J Med Sci. 2019;7:4043. doi: 10.4103/sjmms.sjmms_166_16

5. Suzuki RB, Cabral AD, Martins LPA, Speranca MA. A highly sensitive Leishmania infantum chagasi isolation method from bone marrow and peripheral blood of adults and children. J Infect Dev Ctries. 2016;10(11):1275-1277. doi: 10.3855 /jidc. 8022

6. Abass E, Kang C, Martinkovic F, Semiao-Santos SJ, Sundar $\mathrm{S}$, Walden $\mathrm{P}$, et al. Heterogeneity of Leishmania donovani parasites complicates diagnosis of visceral leishmaniasis: comparison of different serological tests in three endemic regions. PLoS One. 2015;10:e0116408. doi: 10.1371/journal. pone.0116408.
7. Reithinger R. Global burden of cutaneous leishmaniasis. Lancet Infect Dis. 2016;16:1004-1005. doi: 10.1016/S14733099(16)30195-5

8. Akhlagh A, Salehzadeh A, Zahimia AH, Davari B. 10-Year Trends in Epidemiology, Diagnosis, and Treatment of Cutaneous Leishmaniasis in Hamadan Province, West of Iran (2007-2016). Front Public Health. 2019;7:27. doi: 10.3389/ fpubh.2019.00027

9. Ricciardi A, Ndao M. Diagnosis of parasitic infections: what's going on? J Biomol Screen. 2015;20(1):6-21. doi: 10.20546/ijcmas.2018.707.380

10. Trevisan DA, Lonardoni MV, Demarchi IG. Diagnostic methods to cutaneous leishmaniasis detection in domestic dogs and cats. An Bras Dermatol. 2015;90(6):868-872. doi: 10.1590/abd18064841.20153716

11. Galluzzi L, Ceccarelli M, Diotallevi A, Menotta M, Magnani M. Real-time PCR applications for diagnosis of leishmaniasis. Parasite Vector. 2018;11(1):1-13. doi: 10.1186/ s13071-018-2859-8

12. Sagi O, Berkowitz A, Codish S, Novack V, Rashti A, Akad $F$ et al. Sensitive molecular diagnostics for cutaneous leishmaniasis. Open Forum Infect Dis. 2017;4:1-7. doi: 10.1093/ofid/ofx037

13. De Vries HJC, Reedijk SH, Schallig HDFH. Cutaneous Leishmaniasis: recent developments in diagnosis and management. Am J Clin Dermatol. 2015;16:99-109. doi: 10.1007/s40257-015-0114-z

14. Piasecki J, Waligora M, Dranseika V. Google Search an Additional Source in Systematic Review. Sci Eng Ethics. 2018;24:809-810. doi: 10.1007/s11948-0170010-4

15. Mokhtar IK, Mosa BM, Abdallah MIM. Epidemiological and Serological Study of Leishmaniasis in Najran Region, Saudi Arabia. J Biol Life Sci. 2017;8(1):59-71.

16. JamilL A, Zafer M, AL-Fifi S, AL-Jarie A, AL-Shraim M, Shabana M, et al. Clinical and Pathological Features of Visceral Leishmaniasis in Pediatric Patients, Aseer Province, Southwestern Saudi Arabia. Med J Cairo Univ. 2012;80(2):121-126.

17. Jamil AM, Omer FM, Abdalla SEA, Menshawy NE, Ali $\mathrm{AMAB}$. Comparison of an indirect hemagglutination test and bone marrow aspiration for the diagnosis of visceral leishmaniasis in Aseer area, Saudi Arabia. Egypt J Hematol. 2012;37:88-90.

18. Jack FE, Mateen SA, Parker JA. Visceral Leishmaniasis in Medina Region. Sudan J Pediatr. 2005;7:142-157.

19. Al-Orainey IO, Gasim IY, Singh LM, Ibrahim B, Ukabam OS, Gonchikar D, et al. Visceral Leishmaniasis in Gizan, Saudi Arabia. Ann Saudi Med. 1994;14(5):396-398. doi: 10.5144/0256-4947.1994.396

20. Ibrahim EA, Al-Zahrani MA, Al-Tuwaigri AS, AlShammary FJ, Evans DA. Leishmania infecting man and wild animals in Saudi Arabia. 9. The black rat (Rattus rattus) a probable reservoir of visceral Leishmaniasis in Gizan province, south-west Saudi Arabia. Trans R Soc Trop Med Hyg. 1992;86(5):513-514. doi: 10.1016/00359203(92)90090-y

21. Hawash YA, Ismail KA, Abdel-Wahab MM, Khalifa M. Diagnosis, Treatment and clinical features of cutaneous leishmaniasis in Saudi Arabia. Korean J Parasitol. 2018;56(3):229-236. doi: 10.3347/kjp.2018.56.3.229

22. Alahmad Z, Al-Hadi M, Alaithan M, Ashour D. The prevalence of cutaneous leishmaniasis in Al-Ahssa, Saudi Arabia. Int J Adv Educ Res. 2018;3(5): 22-24. 
23. Haouas N, Amer O, Alshammri FF, Al-Shammari S, Remadi L, Ashankyty I. Cutaneous leishmaniasis in northwestern Saudi Arabia: identification of sand fly fauna and parasites. Parasite Vector. 2017;10(1):544. doi: 10.1186/s13071-017-2497-6

24. Barradah RK. Incidence trend of cutaneous leishmaniasis (CL) in Majmaah, Kingdom of Saudi Arabia. Majmaah J Health Sci. 2017;5(2):57-65.

25. Alanazi AD, Alyousif MS, Saifi MA, Alanazi IO. Epidemiological studies on cutaneous leishmaniasis in Ad-Dawadimi District, Saudi Arabia. Trop J Pharm Res. 2016;15(12):2709-2712.

26. Faraj TK, Lake IR. The seasonality of cutaneous leishmaniasis in Asir Region, Saudi Arabia. Int J Environ Sustain. 2014;3(3):1-13.

27. El-Beshbishy HA, Al-Ali KH, El-Badry AA. Molecular characterization of cutaneous leishmaniasis in AlMadinah Al-Munawarah province, western Saudi Arabia. Int J Infect Dis. 2013;17:e334-8. doi: 10.1016/j. exppara.2013.02.016

28. Uthman MAE, Satir AA, Tabbara. Clinical and histological features of zoonotic cutaneous leishmaniasis in Saudi Arabia. J Eur Acad Dermatol Venereol. 2005;19:431-436.

29. Al-zahrani MA, Peters W, Evans DA, Smith V, Ching Chin I. Leishmania infecting man and wild animals in Saudi Arabia 6. Cutaneous leishmaniasis of man in the South-West. Trans R Soc Trop Med Hyg. 1989;83:621-628. doi: 10.1016/0035-9203(89)90376-3

30. Dye C, Killick-Kendrick R, Ben Ismail R, Al-Gindan Y. Zoonotic cutaneous leishmaniasis in Saudi Arabia: Results of a preliminary epidemiological survey in AlAhsa oasis. Trans R Soc Trop Med Hyg. 1989;83:493-498. doi: 10.1016/0035-9203(89)90265-4

31. Gonzalez U, Pinart M, Sinclair D, Firooz A, Enk C, Velez ID, et al. Vector and reservoir control for preventing leishmaniasis. CochraneDatabaseSystRev.2015;CD008736. doi: 10.1002/14651858.CD008736.pub2.
32. Cutaneous Leishmaniasis Management Guide. National Ministry of Health, Public Health Deputyship, Saudi Arabia. 2019. (Accessed October 21, 2019). https://www. moh.gov.sa/en/Ministry/MediaCenter/Publications/ Documents / National-Policy-for-Management-ofCutaneous-Leishmaniasis-Cases.pdf

33. Vallur AC, Reinhart C, Mohamath R, Goto Y, Ghosh P, Mondal D, et al. Accurate Serodetection of Asymptomatic Leishmania donovani Infection by Use of Defined Antigens. J Clin Microbiol. 2016;54(4):1025-1030. doi: 10.1128/JCM.02620-15

34. Mahamoud A, Osman HA, Semiao-Santos SJ, Harith AE, Abass EM, Agib AE, et al. Identification of an area predominantly endemic for childhood and adolescent visceral leishmaniasis in central Sudan. Acta Tropica. 2018;178:142-147. doi: 10.1016/j.actatropica.2017.11.010

35. Abuzaid AA, Abdoon AM, Aldahan MA, Alzahrani AG Alhakeem RF, Asiri AM, et al. Cutaneous leishmaniasis in Saudi Arabia: a comprehensive overview. Vector Borne Zoonotic Dis. 2017;17:673-684. doi: 10.1089/vbz.2017.2119

\section{Authors' Contribution:}

EA, conceptualization, design, interpretation of data, project administration, supervision, writing and editing.

ZA, methodology, writing manuscript draft. LYZ, checked validity of data, revise and editing of manuscript.
Authors:

1. Elfadil Abass,

2. Zainab Al-Hashem,

3. Lamya Zohair Yamani,

1-3: Department of Clinical Laboratory Science, College of Applied Medical Sciences, Imam Abdulrahman Bin Faisal University, Dammam,

Saudi Arabia. 